\section{Thyroid Dysfunction in Patients with Antineutrophil Cytoplasmic Antibody-associated Vasculitis: A Monocentric Retrospective Study}

To the Editor:

Antineutrophil cytoplasmic antibody (ANCA)-associated vasculitis (AAV) is characterized by necrotizing vasculitis in small-sized vessels such as arterioles, capillaries, and venules ${ }^{1}$. The possibility of a link between thyroid dysfunction and autoimmunity has been considered and its prevalence was reported differently according to each autoimmune disease ${ }^{2}$. Given that AAV is one of the systemic autoimmune diseases affecting most major organs ${ }^{3}$, and there is cross-reactivity between thyroid peroxidase and myeloperoxidase (MPO) molecules ${ }^{4}$, the prevalence of thyroid dysfunction, including autoimmune thyroiditis, may be increased in patients with AAV. Previous studies reported the higher prevalence of thyroid dysfunction in patients with AAV than in the general population ${ }^{5,6,7}$. However, there was no study on the prevalence of thyroid dysfunction in AAV patients in Korea. In this study, we investigate the prevalence of thyroid dysfunction and searched for

Table 1. Comparison of variables between patients with and without thyroid dysfunction.

\begin{tabular}{|c|c|c|c|}
\hline Variables & $\begin{array}{c}\text { Patients without } \mathrm{P} \\
\text { Thyroid Dysfunction, } \\
\mathrm{n}=159\end{array}$ & $\begin{array}{l}\text { Patients with Thyroid } \\
\text { Dysfunction, } \\
n=27\end{array}$ & $\mathrm{p}$ \\
\hline \multicolumn{4}{|l|}{ Demographics } \\
\hline Age at diagnosis, yrs & $55.7 \pm 14.9$ & $57.9 \pm 1.8$ & 0.464 \\
\hline Male sex & $51(32.1)$ & $6(22.2)$ & 0.305 \\
\hline Variants of AAV & & & 0.139 \\
\hline MPA & $80(50.3)$ & $19(70.4)$ & \\
\hline GPA & $41(25.8)$ & $5(18.5)$ & \\
\hline EGPA & $38(23.9)$ & $3(11.1)$ & \\
\hline \multicolumn{4}{|l|}{ ANCA positivity at diagnosis } \\
\hline MPO-ANCA (or P-ANCA) & $97(61.0)$ & $22(81.5)$ & 0.040 \\
\hline PR3-ANCA (or C-ANCA) & $26(16.4)$ & $4(14.8)$ & 0.841 \\
\hline MPO-ANCA (or P-ANCA) and PR3-ANCA & & & \\
\hline (or C-ANCA) & $6(3.8)$ & $2(7.4)$ & 0.390 \\
\hline ANCA-negative & $42(26.4)$ & $3(11.1)$ & 0.086 \\
\hline \multicolumn{4}{|l|}{ Clinical manifestations at diagnosis } \\
\hline General & $71(44.7)$ & $12(44.4)$ & 0.984 \\
\hline Cutaneous & $33(20.8)$ & $8(29.6)$ & 0.304 \\
\hline Mucous membranes and eye & $8(5.0)$ & $4(14.8)$ & 0.056 \\
\hline Ear, nose, and throat & $65(40.9)$ & $6(22.2)$ & 0.065 \\
\hline Pulmonary & $88(55.3)$ & $16(59.3)$ & 0.705 \\
\hline Cardiovascular & $39(24.5)$ & $9(33.3)$ & 0.334 \\
\hline Gastrointestinal & $10(6.3)$ & $0(0)$ & 0.180 \\
\hline Renal & $89(56.0)$ & $20(74.1)$ & 0.077 \\
\hline Nervous systemic & $52(32.7)$ & $6(22.2)$ & 0.277 \\
\hline \multicolumn{4}{|c|}{ Vasculitis activity and prognostic factors at diagnosis } \\
\hline BVAS & $12.5 \pm 7.1$ & $13.8 \pm 6.9$ & 0.381 \\
\hline 2009 FFS & $1.3 \pm 1.0$ & $1.6 \pm 1.0$ & 0.074 \\
\hline Followup duration, mos & $49.4 \pm 48.9$ & $51.6 \pm 47.0$ & 0.824 \\
\hline \multicolumn{4}{|l|}{ Comorbidities at diagnosis and during followup } \\
\hline CKD stage $3,4,5$ & $36(22.6)$ & $17(63.0)$ & $<0.001$ \\
\hline Endstage kidney disease & $26(16.4)$ & $2(7.4)$ & 0.229 \\
\hline Diabetes mellitus & $32(20.1)$ & $6(22.2)$ & 0.803 \\
\hline Hypertension & $64(40.3)$ & $16(59.3)$ & 0.065 \\
\hline Interstitial pneumonia & $7(4.4)$ & $10(37.0)$ & 0.498 \\
\hline Ischemic heart disease & $7(4.4)$ & $5(18.5)$ & 0.006 \\
\hline Cardiovascular or cerebrovascular accident & $16(10.1)$ & $0(0)$ & 0.085 \\
\hline \multicolumn{4}{|l|}{ Immunosuppressive drugs during followup } \\
\hline Glucocorticoids & $137(86.2)$ & $25(92.6)$ & 0.357 \\
\hline Cyclophosphamide & $64(40.3)$ & $14(51.9)$ & 0.259 \\
\hline Rituximab & $16(10.1)$ & $4(14.8)$ & 0.461 \\
\hline Azathioprine & $52(32.7)$ & $10(37.0)$ & 0.659 \\
\hline Mycophenolate mofetil & $12(7.5)$ & $0(0)$ & 0.140 \\
\hline Tacrolimus & $8(5.0)$ & $1(3.7)$ & 0.766 \\
\hline Methotrexate & $13(8.2)$ & $1(3.7)$ & 0.415 \\
\hline
\end{tabular}

Values are mean \pm SD or $\mathrm{n}(\%)$. ANCA: antineutrophil cytoplasmic antibody; AAV: ANCA-associated vasculitis; MPA: microscopic polyangiitis; GPA: granulomatosis with polyangiitis; EGPA: eosinophilic GPA; MPO: myeloperoxidase; P: perinuclear; PR3: proteinase 3; C: cytoplasmic; BVAS: Birmingham Vasculitis Activity Score; FFS: 5-factor score; CKD: chronic kidney disease. 
the predictors at diagnosis of its development during followup for 3 months or greater in Korean patients with AAV.

We retrospectively reviewed the medical records of 186 patients with AAV, who were classified as having AAV at the Department of Rheumatology, Yonsei University College of Medicine, Severance Hospital, from October 2000 to July 2018. They met the 2007 European Medicines Agency algorithms and the 2012 Chapel Hill Consensus Conferences Nomenclature of Vasculitis ${ }^{1,8}$. All patients had well-documented medical records regarding both AAV and thyroid diseases. This study was approved by the Institutional Review Board (IRB) of Severance Hospital (4-2017-0673), and patient written informed consent was waived by the approving IRB, because this was a retrospective study.

The followup duration was defined for patients with thyroid dysfunction as the period from diagnosis to development, and as the period from diagnosis to the last visit for those without thyroid dysfunction. Overt thyroid dysfunction was approved by the International Classification Diseases, 10th revision, or by medications searched through the Korean Drug Utilisation Review system. Subclinical hyperthyroidism was defined when a thyroid- stimulating hormone (TSH) level is suppressed, but a triiodothyronine (T3) level is within a normal range, whereas subclinical hypothyroidism was defined when a TSH level is enhanced but a free thyroxine (T4) level is within a normal range ${ }^{9}$. Differences in variables between the 2 groups were analyzed using the chi-square and Fisher's exact tests or the Mann-Whitney U test. Comparison of cumulative thyroid dysfunction-free survival between the 2 groups was analyzed by the Kaplan-Meier survival analysis. P values $<0.05$ were considered statistically significant.

Twenty-seven of 186 patients (14.5\%) exhibited thyroid dysfunction, and 7 of them had overt thyroid dysfunction before diagnosis of AAV (Supplementary Table 1, available from the authors on request). The overall prevalence was lower than in previous studies (20.0-21.5\%), but similar to that of another previous study $(14.5 \%)^{5,6,7}$. Based on the report on thyroid dysfunction in the Korean general population ${ }^{10}$, patients with AAV exhibited the significantly increased prevalences of overt hyperthyroidism and hypothyroidism compared to the general population (5.4\% vs $0.5 \%$ and $4.3 \%$ vs $0.7 \%$, respectively). Also, patients with AAV exhibited a lower prevalence of subclinical hyperthyroidism than the general population (1.6\% vs $3.0 \%$ ) but not subclinical hypothyroidism (3.2\% vs $3.1 \%)$.

When we divided $186 \mathrm{AAV}$ patients into the 2 groups according to the presence of thyroid dysfunction, we found that patients with thyroid dysfunction had MPO-ANCA [or perinuclear (P)-ANCA] at diagnosis more frequently than those without ( $81.5 \%$ vs $61.0 \%)$. During followup, chronic kidney disease (stages 3-5) and ischemic heart disease were observed in patients with thyroid dysfunction more commonly than in those without, which could suggest that thyroid dysfunction might influence the development of chronic kidney disease or ischemic heart disease. Immunosuppressive drugs were evenly administered to both groups (Table 1).

In the analysis of the development of thyroid dysfunction after AAV diagnosis, we divided 179 AAV patients into 2 groups according to each variable at diagnosis and searched for the predictors. Patients with MPO-ANCA (or P-ANCA) and the 2009 5-factor score $\geq 2$ at diagnosis exhibited the lower cumulative thyroid dysfunction-free survival than those without during followup (Figure 1). Age $\geq 65$ years, ANCA positivity, and renal involvement at diagnosis, but not sex, were also predictors of thyroid dysfunction (Supplementary Figure 1, available from the authors on request). It was reported that propylthiouracil (PTU) could induce AAV development, particularly in women with renal involvement ${ }^{6}$. In this study, because only 1 patient received PTU 8 months before AAV diagnosis, we could not clarify it.

Our study has several limitations of retrospective and monocentric study designs. The number of patients was not large enough to represent all Korean patients with AAV and no requalified results were available in all patients. However, this study is a pilot study to first discover the prevalence and the predictors at diagnosis of thyroid dysfunction in Korean patients with AAV. In the future, prospective studies with a larger number of patients and the serial results of thyroid function test will provide more reliable and validated information on the clinical implication of thyroid dysfunction in patients with AAV.

JAE YEON KIM, Department of Medicine, Yonsei University College of Medicine; YONG-BEOM PARK, MD, PhD; SANG-WON LEE D, MD, PhD, Division of Rheumatology, Department of Internal Medicine, Yonsei University College of Medicine, and Institute for Immunology and Immunological Diseases, Yonsei University College of Medicine, Seoul, South Korea. This study was supported by a faculty research grant of Yonsei University College of Medicine (6-2016-0145) and a grant from the Korea Health Technology R\&D Project through the Korea Health Industry Development Institute, funded by the Ministry of Health and Welfare, Republic of Korea (HI14C1324). Address correspondence to Prof. S.W. Lee, Division of Rheumatology, Department of Internal Medicine, and Institute for Immunology and Immunological Diseases, Yonsei University College of Medicine, 50-1 Yonsei-ro, Seodaemun-gu, Seoul 03722, South Korea. E-mail: sangwonlee@yuhs.ac

\section{REFERENCES}

1. Jennette JC, Falk RJ, Bacon PA, Basu N, Cid MC, Ferrario F, et al. 2012 revised International Chapel Hill Consensus Conference Nomenclature of Vasculitides. Arthritis Rheum 2013;65:1-11.

2. Santoro D, Vadalà C, Siligato R, Buemi M, Benvenga $S$. Autoimmune thyroiditis and glomerulopathies. Front Endocrinol $2017 ; 8: 119$

B

A

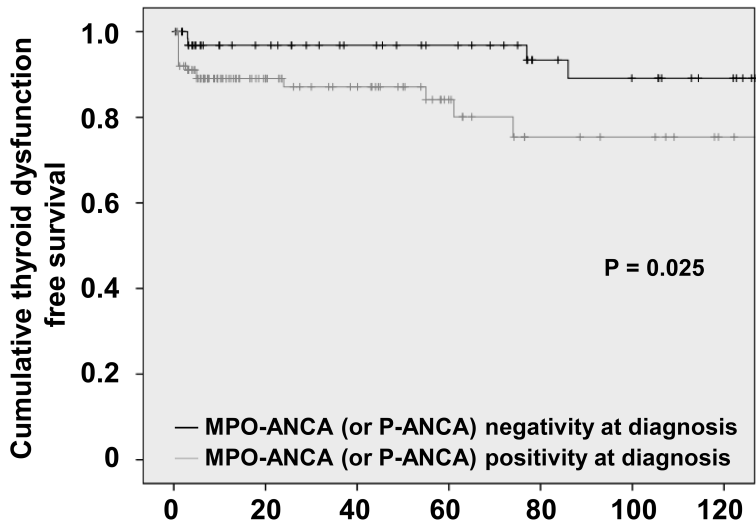

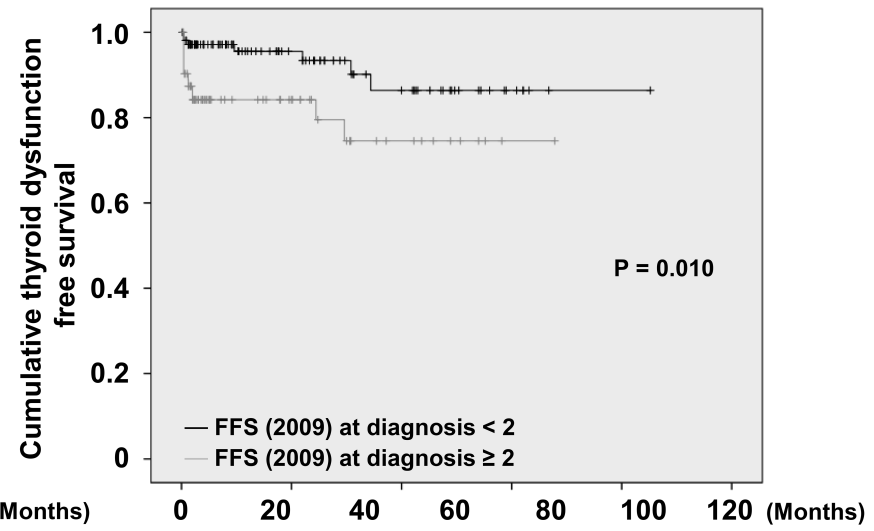

Figure 1. Predictors at diagnosis of thyroid dysfunction development. MPO-ANCA (or P-ANCA) positivity and 2009 FFS $\geq 2$ at diagnosis were the predictors of the development of thyroid dysfunction after diagnosis. MPO: myeloperoxidase; ANCA: antineutrophil cytoplasmic antibody; P: perinuclear; FFS: 5-factor score.

Personal non-commercial use only. The Journal of Rheumatology Copyright $\subset$ 2019. All rights reserved. 
3. Millet A, Pederzoli-Ribeil M, Guillevin L, Witko-Sarsat V, Mouthon L. Antineutrophil cytoplasmic antibody-associated vasculitides: is it time to split up the group? Ann Rheum Dis 2013;72:1273-9.

4. Haapala AM, Hyöty H, Parkkonen P, Mustonen J, Soppi E. Antibody reactivity against thyroid peroxidase and myeloperoxidase in autoimmune thyroiditis and systemic vasculitis. Scand J Immunol 1997;46:78-85.

5. Prendecki M, Martin L, Tanna A, Antonelou M, Pusey CD. Increased prevalence of thyroid disease in patients with antineutrophil cytoplasmic antibodies-associated vasculitis. J Rheumatol 2018;45:686-9.

6. Lionaki S, Hogan SL, Falk RJ, Joy MS, Chin H, Jennette CE, et al. Association between thyroid disease and its treatment with ANCA small-vessel vasculitis: a case-control study. Nephrol Dial Transplant 2007;22:3508-15.

7. Englund M, Merkel PA, Tomasson G, Segelmark M, Mohammad AJ. Comorbidities in patients with antineutrophil cytoplasmic antibody-associated vasculitis versus the general population. J Rheumatol 2016;43:1553-8.
8. Watts R, Lane S, Hanslik T, Hauser T, Hellmich B, Koldingsnes W, et al. Development and validation of a consensus methodology for the classification of the ANCA-associated vasculitides and polyarteritis nodosa for epidemiological studies. Ann Rheum Dis 2007;66:222-7.

9. Surks MI, Ortiz E, Daniels GH, Sawin CT, Col NF, Cobin RH, et al. Subclinical thyroid disease: scientific review and guidelines for diagnosis and management. JAMA 2004;291:228-38.

10. Kim WG, Kim WB, Woo G, Kim H, Cho Y, Kim TY, et al. Thyroid stimulating hormone reference range and prevalence of thyroid dysfunction in the Korean population: Korea National Health and Nutrition Examination Survey 2013 to 2015. Endocrinol Metab 2017;32:106-14.

J Rheumatol First Release May 15 2019; 2019;46:9;

doi:10.3899/jrheum.181270 